\title{
Automatic Search of Nursing Diagnoses
}

\author{
Matías A. Morales ${ }^{1}$, Rosa L. Figueroa ${ }^{1}$, and Jael E. Cabrera ${ }^{2}$ \\ ${ }^{1}$ Electrical Engineering Department, University of Concepción, Chile \\ ${ }^{2}$ Cardiac Surgery Department, Guillermo Grant Benavente Hospital, \\ Cardiac Surgery ICU, Chile
}

\begin{abstract}
Nursing documentation is all the information that nurses register regarding the clinical assessment and care of a patient. Currently, these records are manually written in a narrative style; consequently, their quality and completeness largely depends on the nurse's expertise. This paper presents an algorithm based on standardized nursing language that searches and sorts nursing diagnoses by its relevance through a ranking. Diagnoses identification is performed by searching and matching patterns among a set of patient needs or symptoms and the international standard of nursing diagnoses NANDA. Three sorting methods were evaluated using 6 utility cases. The results suggest that TF-IDF ( $83.43 \%$ accuracy) and assignment of weights by hit $(80.73 \%$ accuracy) are the two best alternatives to implement the ranking of diagnoses.
\end{abstract}

Keywords: NANDA, Nursing documentation, Pattern Matching, Diagnosis retrieval, Decision Support, TF, TF-IDF.

\section{Introduction}

In nursing all information concerning the care and management of patients is documented in the form of patient records. In many places this information is stored manually in paper records, so its quality and completeness is determined by the nurse's expertise. Currently, there are standards and terminology available that could be used to facilitate the transition to electronic records. Among them we find the terminology established by the North American Nursing Diagnosis Association (NANDA) [1]. This terminology has a total of 188 nursing diagnoses including label, code, definition, defining characteristics, risk factors, and related factors.

In this paper, we present an algorithm that was developed and implemented as part of a nursing care software prototype. The system identifies and retrieves diagnoses from a set of needs that are identified in the clinical assessment of the patient according to the nursing model of Virginia Henderson [2].

\section{Materials and Methods}

\subsection{Data Set}

Two data sources were used by our algorithm: NANDA databases and a patient assessment chart. NANDA databases in Spanish were created and are being populated 
by nurses of the Complejo Hospitalario de Jaén ${ }^{1}$. The patient assessment chart used in this work was created by the researchers in the first stage of the project and is based on Virginia Henderson's model. It contains a set of needs and symptoms commonly used by nurses in the cardio-surgery intensive care unit (ICU) to evaluate their patients.

\subsection{Search and Identification of Nursing Diagnoses Algorithm}

We implemented a pattern matching algorithm that searches for similarities between a set of needs or symptoms that are obtained from the clinical assessment of the patient and the available information in the NANDA database. Every need is searched among the information that describes each NANDA diagnosis (defining characteristics, related factors and risk factors). In order to improve the search and identification of patterns a tailored medical synonym dictionary was created and used within the search. Abbreviations were expanded and all the texts were normalized, i.e. letters were converted to lower case and accent marks and other diacritics were removed. Some special characters were not eliminated since they were thought to be important for the search (example: temperature is commonly referred as $\mathrm{T}^{\circ}$ ).

From pattern matching we obtained a co-occurrence matrix where the columns corresponded to the needs or symptoms of the patient and the rows to the NANDA diagnoses. Then, each diagnosis $d_{j}$ can be represented by a weight vector $d_{j}=$ $\left\langle\mathrm{w}_{\mathrm{j} 1}, \ldots, \mathrm{w}_{\mathrm{jn}}\right\rangle$, where $\mathrm{n}$ is the total number of different needs across the whole assessment chart, and $w_{j k}$ indicates the importance of the $t_{k}$ need in $d_{j}$ [3]. This matrix is used for the next step which will define the relevance ranking of the retrieved list of diagnoses.

\subsection{Diagnoses Relevance Ranking}

We studied and compared three methods to create a diagnoses relevance ranking. These methods worked by assigning weights to each diagnosis according to the information contained on the co-occurrence matrix.

Frequency of the Terms (T.F.). This method assigns weights based on the frequency of co-occurrence of symptoms or needs in the defining characteristics, related factors and risk factors for each diagnosis. Frequencies of all the different symptoms considered in the search are added and the resulting score is assigned to that diagnosis. Once scores are assigned, diagnoses are sorted in a descending order.

Assignment of Weights by Hit or Coincidence. Each time one of the searched symptoms appears in the information related to the diagnoses $\left(f_{j k}>0\right)$ a hit or match is marked [4]. Once the hits for each diagnosis are detected, scores are assigned by adding the hits of those symptoms. Unlike the previous method, this one identifies the presence or absence of the search words, thus the score given to the diagnosis will depend of the presence or absence of the symptom and not on the number of times that this symptom shows in a given diagnosis.

\footnotetext{
${ }^{1}$ Nursing Jaen, http://www.gratisweb.com/enferjoja/
} 
Weights Assignment Using TF-IDF. The I.D.F. method [5] calculates the weight of every need or symptom according to the inverse value of its frequency in the set of diagnoses. Thus, the IDF factor of a need or symptom $t_{k}$ is given by:

$$
i d f\left(t_{k}\right)=\log \left(\frac{N}{d f\left(t_{k}\right)}\right) .
$$

Where, $N$ is the total number of diagnoses and $d f\left(t_{k}\right)$ is the number of diagnoses that contain $\mathrm{t}_{\mathrm{k}}$.

TF-IDF calculates the weight of every need or symptom in the vector that represents the diagnosis by taking into account the inverse frequency of the term in the diagnosis, combining it with the frequency of the term within each document (Eq. 2):

$$
w_{j k}=t f\left(t_{k}, d_{j}\right) \cdot i d f\left(t_{k}\right)
$$

In order to solve the equation above, we first calculate the frequency of the need or symptom $t_{k}$ in the diagnosis $d_{j}(T F)$. Then, we calculate the inverted frequency of the diagnosis $d_{j}$ using Eq.1. Finally, both measures are combined using Eq.2. Scores for every considered symptom are added to obtain the score for the diagnosis.

Table 1 shows an example for two conditions: "arrhythmias" and "edema". As the arrhythmias is only present in the diagnosis 1 and 3, the $d f$ of arrhythmias is $d f_{a}=2$, and as there are in total 188 NANDA diagnoses, $N=188$, the inverse frequency of the arrhythmia diagnosis is $i d f_{a}=\log (188 / 2)=1.97$; "Edema" is present in the 3 diagnoses, thus its $d f_{a}=3$, then inverse frequency of the edema diagnosis is $i d f_{e}=\log (188 / 3)=1.79$. Once TF and IDF values are obtained, the weight of each symptom within each diagnosis is determined by multiplying the frequency of the symptom by its inverse frequency resulting in the weight of the symptom. Finally, a general score for a given diagnosis is determined as the sum of the weights obtained by each symptom. In Table 1 the given order is: diagnosis 2, diagnosis 1, diagnosis 3 .

Table 1. Scores calculation examples of Weight assignment by Hit

\begin{tabular}{llll}
\hline Diagnostics & Arrhythmias & Edema & Score \\
\hline Diagnosis 1 & 2 & 1 & $1.97 * 2+1.79 * 1=5.73$ \\
Diagnosis 2 & 0 & 7 & $1.97 * 0+1.79 * 7=12.53$ \\
Diagnosis 3 & 1 & 1 & $1.97 * 1+1.79 * 1=3.76$ \\
\hline
\end{tabular}

\subsection{Diagnosis Ranking Assessment}

Evaluation of the algorithm was performed in 6 examples of use. These examples were reviewed by a nurse who determined which diagnoses would be expected given a certain set of symptoms and needs. To evaluate the ranking of retrieved diagnoses we compared the list of diagnoses given by the nurse with the results obtained by the three methods described in Section 2.3. 
To estimate effectiveness of document retrieval tools, two measures are usually used [6]: Precision and Recall. The Recall measures the proportion of documents - in this case relevant diagnoses - that the system is able to retrieve. Precision measures the proportion of the recovered diagnoses that are relevant to the query. In this case the metric we used to evaluate and compare the rankings is the mean average precision (MAP) which is based on the metrics discussed earlier.

MAP is the average of the non-interpolated average precision over all queries [6]:

$$
\operatorname{MAP}(Q)=\frac{1}{|Q|} \sum_{j=1}^{|Q|} \frac{1}{m} \sum_{j=1}^{m} \operatorname{Precision}\left(R_{j k}\right) .
$$

Where,
$Q \quad$ : Total number of consultations.
$j \quad$ : Index of consultation.
$m \quad$ : Total number of relevant documents.
$k \quad$ : Top k of documents.
$R \quad$ : Documents retrieved.

MAP is suitable for the evaluation of rankings because it rewards methods that provide high scores to the relevant documents; that is, sorted into the top places in the list.

Since the system can retrieve up to the top 15 of diagnoses for each of the 6 cases, the assessment was made with the Top-5, Top-10 and Top-15. Only the first 15 diagnoses were evaluated, as it is expected that the algorithm delivers the relevant diagnoses within the first listed.

\section{Results}

Table 2 presents MAP score results. It is observed that the MAP score tends to decrease as the number of documents to be evaluated increase; that is, going from Top-5 to Top-15. It can be noted that for each case both "Assignment of Weights by Hit" and "TF-IDF" have higher MAP values than the "TF" method.

The method "TF-IDF" has a better response in finding the first documents (MAP = 0.9625), which makes it a very efficient method compared to the "TF" method (MAP $=0.7366)$. The second best method is the "Assigning Weight by Hit" (MAP = 0.95). In fact, MAP values for "TF-IDF" are very similar to the MAP values obtained by the "Assignment of Weights by Hit". This can be explained by the way in which both methods retrieve the diagnoses. For instance, with "TF-IDF" for each of the 6 queries the first two diagnoses corresponded to those expected by the nurse. In the case of the "Assignment of Weights by Hit", 4 of the 6 queries retrieved relevant diagnoses within the first two positions of the list. In the 2 remaining consultations the expected results were within the Top-5, but not necessarily in the first or second position. 
Table 2. MAP comparison for different number of documents

\begin{tabular}{cccc}
\hline $\begin{array}{c}\text { Number of } \\
\text { Documents }\end{array}$ & MAP TF & $\begin{array}{c}\text { MAP } \\
\text { Weight by Hit }\end{array}$ & $\begin{array}{c}\text { MAP TF- } \\
\text { IDF }\end{array}$ \\
\hline 5 & 0.7366 & 0.95 & 0.9625 \\
10 & 0.7121 & 0.85 & 0.8769 \\
15 & 0.6995 & 0.8073 & 0.8343 \\
\hline
\end{tabular}

\section{Conclusions}

This article presents a nursing diagnoses search algorithm that uses a set of needs and symptoms from the clinical assessment of a patient. Six use cases were taken as base for the evaluation of the search algorithm and the relevance ranking of diagnoses.

Results suggest a reasonable performance of the algorithm for the identification and recovery of diagnoses (83.43\% accuracy Top-15 for TF-IDF, $80.73 \%$ accuracy Top 15 for "Assignment of Weight by Hit"). We noted that the performance of the algorithm may have been affected by the lack of data on the Spanish version of the NANDA databases used but even with the available data the results are reasonable and promising for future developments that consider a more extensive evaluation of both usability and accuracy. Because of its simplicity, relevance ranking of diagnoses based on "Assignment of Weights by Hit" was chosen to implement the software prototype for the nursing care process.

The methods shown in this paper allow for automatically retrieving a list of nursing diagnoses. This system constitutes a support tool for the nursing process that assists in the assessment and/or evaluation of the patient and suggests possible diagnoses, but the final decision of the diagnosis is left to the nurse or care giver.

\section{Future Work}

We propose to implement manual search of NANDA diagnosis by name or code that will allow the nurse to find diagnoses directly. Also, since this is just a preliminary study we plan to perform an extended evaluation involving more use cases. Finally, an interesting future work will be to evaluate the impact of the system in a clinical environment.

Acknowledgments. The authors would like to thank the professional nurse José Jaen of the Jaén Hospital Complex for facilitating the NANDA databases in Spanish.

\section{References}

1. NANDA International. Diagnósticos Enfermeros: Definiciones y Clasificación (2008)

2. Caballero, M.E., Becerra, S.R., Hullin, L.C.: Proceso de Enfermería e Informática (2010) 
3. Zuñiga Cuevas, R.E.: Codificación de Diagnósticos Médicos utilizando técnicas de aprendizaje automático (2010)

4. Grossman, D.A., Frieder, O.: Information Retrieval, Algorithms and Heuristics (2004)

5. Manning, C.D., Raghavan, P., Schütze, H.: An Introduction to Information Retrieval (2009)

6. Tolosa, G.H., Bordignon, F.: Introduction to Information Retrieval: Concepts, models and basic algorithms 